\title{
Immunotherapy with check-point inhibitors (CPI) in adult malignancies: a protocol for the systematic review of the quality of economic analyses
}

\author{
Ying Wang ${ }^{1 *}$, Pierre Camateros ${ }^{2}$, Denise Smith $^{3}$, David Dawe ${ }^{4}$ and Peter Ellis ${ }^{5}$
}

\begin{abstract}
Background: Immuno-oncology, and in particular, check-point inhibitors (CPIs), have led to a paradigm shift in the field of cancer care. The cost of new drug development is high, and many novel agents in oncology are significantly more expensive than older agents. Therefore, healthcare funders have factored measures of costeffectiveness into decisions concerning drug reimbursement and incorporation of new agents into treatment algorithms. The methodology of cost-effectiveness evaluations, however, is less rigorously applied than those evaluating clinical efficacy and safety data. Thus, in spite of many regulatory bodies having approved CPIs based on existing economic analyses, to date, there has not been a systematic evaluation of the quality of health economic studies conducted on this new class of agents.

Therefore, we propose to systematically review the methodologic and reporting quality of cost-effectiveness and cost-utility studies assessing CPIs to alternate established therapies, other immuno-oncology regimens, or placebo, in adults with malignancies.

Methods/design: The systematic review will include all published economic evaluations of CPIs compared with at least one other treatment in adult patients with solid or hematologic malignancies. A search will be performed to identify relevant studies in Ovid MEDLINE, EMBASE, Cost-effectiveness Analysis Registry, Evidence-Based Medicine Reviews, and the NIHR-HTA database. The titles and abstracts of all identified studies will be independently reviewed by two reviewers, who will then assess the full text of all articles deemed to meet eligibility criteria. Assessed articles will be screened for compliance with the Consolidated Health Economic Evaluation Reporting Standards (CHEERS) criteria. The association, with CHEERS criteria, of the journal impact factor, publication year, funding source, tumor site, trial or model-based study, and CPIs studied, will then be assessed.
\end{abstract}

Discussion: The systematic review will aim to provide an overview of the quality of economic analyses evaluating CPIs for the treatment of malignancies in adult patients. Any systemic or recurrent deficiencies in methodological or reporting quality will be described and used to inform recommendations for improved reporting of economic analyses.

Systematic review registration: This review will not be registered with PROSPERO, it does not meet the eligibility criterion of addressing an outcome of the direct patient or clinical relevance.

Keywords: Immuno-therapy, Immune-oncology, Check-point inhibitor, Economic analysis, Cost-effectiveness, Cancer, Neoplasm, Quality, Reporting quality, Protocol, CHEERS criteria

\footnotetext{
* Correspondence: ying.wang@alumni.ubc.ca

'BC Cancer Vancouver, 600 West 10th Ave, Vancouver, British Columbia V5Z

4E6, Canada

Full list of author information is available at the end of the article
}

(c) The Author(s). 2019 Open Access This article is distributed under the terms of the Creative Commons Attribution 4.0 International License (http://creativecommons.org/licenses/by/4.0/), which permits unrestricted use, distribution, and reproduction in any medium, provided you give appropriate credit to the original author(s) and the source, provide a link to the Creative Commons license, and indicate if changes were made. The Creative Commons Public Domain Dedication waiver (http://creativecommons.org/publicdomain/zero/1.0/) applies to the data made available in this article, unless otherwise stated. 


\section{Background Rationale}

Immuno-oncology has led to a paradigm shift in the field of cancer care. Since its inception, this new class of systemic cancer therapy has received at least 26 Food and Drug Administration (FDA) approvals across 17 different cancer types, with 940 more agents being studied in clinical development as of September 2017 [1].

Contrary to traditional cytotoxic chemotherapy, CPIs enhance the body's immune system to induce antitumor activity [2]. This allows for comparatively more tolerable adverse events and increased efficacy in certain tumor types. CPIs have been integrated within the treatment pathways of many solid and hematologic malignancies, such as lung and head and neck cancer, urothelial and renal cell carcinoma, and lymphoma. Furthermore, CPIs are under development for use in many more tumor types.

Since healthcare in countries such as Canada and the UK is administered under a publicly funded system, governments have insisted that, prior to approval for funding, new therapies must not only demonstrate clinical effectiveness, but also cost-effectiveness as well. Countries without single-payer systems are also facing the need to account for the rising costs of care. The American Society of Clinical Oncology (ASCO) has recently focused attention on the importance of considering financial impacts in addition to other criteria when assessing new therapies [3]. Thus, along with clinical effectiveness, evaluations of the economic effectiveness of immunotherapies, and in particular check-point inhibitors (CPIs), is of importance.

Unlike the validated approaches for the assessment of clinical effectiveness, such as randomized controlled trials, the assessment of cost-effectiveness is currently not as rigorously scrutinized. Some analyses combine realworld patient-level data with quality of life data collected from randomized controlled trials in single disease sites [4]. Other studies combine multiple disease sites across different countries with inherently different perspectives and healthcare payment systems [5, 6]. Decision-makers often use incremental cost-effectiveness ratios (ICERs) to compare new immunotherapy regimens with existing therapies; however, this method is nuanced and plagued by inaccuracies, such that experts in the field are calling into question the true validity of such analyses [7]. Others have proposed the addition of a standardized scale for grading financial toxicity, parallel to the Common Terminology Criteria for Adverse Events, a system commonly used to evaluate new agents in clinical trials [8].

In an attempt to standardize health economic studies, the International Society of Pharmacoeconomics and Outcomes Research (ISPOR) has developed an aggregate guideline for authors and reviewers to take into consideration. The Consolidated Health Economic Evaluation Reporting Standards (CHEERS) Statement was developed by international experts, and jointly endorsed by 10 journals including the British Medical Journal in $2013[9,10]$.

Previous studies have adopted the CHEERS checklist as their reference for evaluating the quality of published health economic studies in multiple settings [11-13]. In particular, the quality of economic analyses of oral cancer drugs [14], multiple myeloma therapies [15], and adjuvant breast cancer radiotherapy [16], have been assessed in this way. These studies have revealed that adherence to the CHEERS standards was quite variable ranging from 50\% to nearly complete compliance.

Although many regulatory bodies have approved immunotherapies based on existing economic analyses, there has not been a review of the quality and reporting of health economic studies conducted on this new class of agents.

\section{Objectives}

Thus, we propose to systematically review the methodologic and reporting quality of cost-effectiveness and cost-utility studies comparing new CPIs used in immuneoncology regimens, including anti-PD1 agents (nivolumab and pembrolizumab), anti-PDL1 agents (atezolizumab, avelumab, and durvalumab), and the anti-CTLA4 agents (ipilimumab and tremelimumab), to established therapies in adults with solid and hematologic malignancies.

\section{Methods}

\section{Protocol and registration}

This review will not be registered with the international prospective register of systematic reviews (PROSPERO) as it does not meet the eligibility criterion of addressing an outcome of direct patient or clinical relevance.

This protocol was developed based on the Preferred Reporting Items for Systematic review and Meta-Analysis Protocols (PRISMA-P) 2015 checklist (Additional file 1: Figure S1) $[17,18]$, and any protocol amendments will be described, dated, and a rationale provided.

\section{Inclusion and exclusion criteria}

Only studies meeting the following criteria will be included:

- Articles reporting on the results of economic analyses with cost-effectiveness, cost-utility, or costbenefit analyses. This includes pre-planned analyses within randomized control trials, meta-analyses, or any study design using appropriate economic modeling. Articles focusing on the methodological aspects of economic analyses or reporting only on cost-of-illness or budget impacts will be excluded.

- Articles comparing at least two treatment arms, one of which could be placebo, in adults (at least 18 years old or older, or if children were included, where the data for treatment in adults is separately 
Table 1 Search strategy

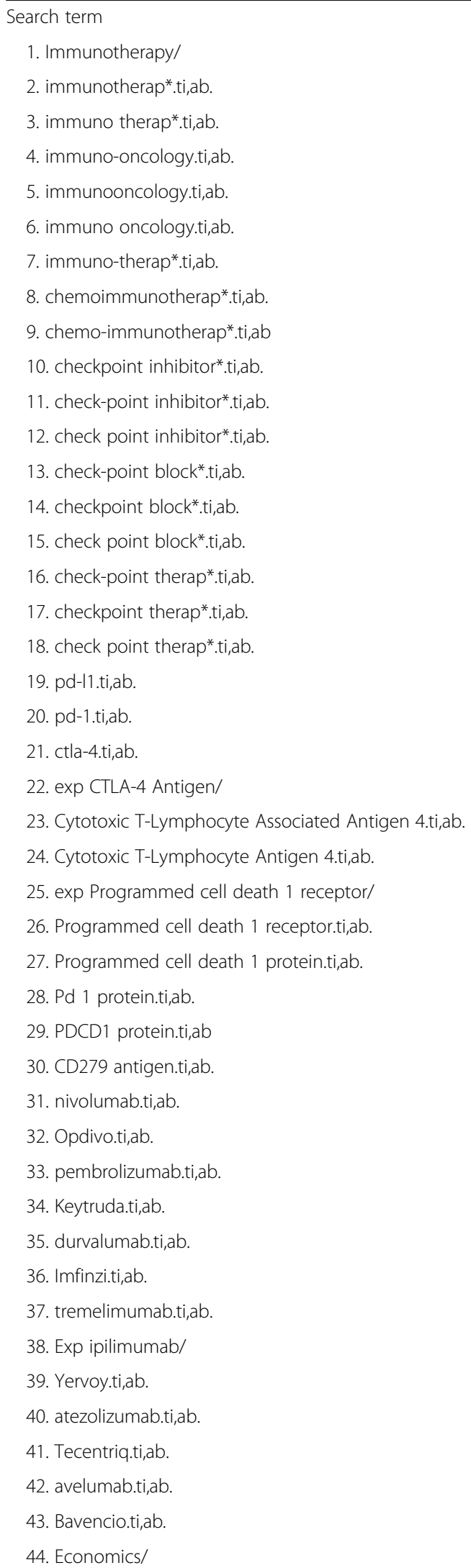

Table 1 Search strategy (Continued)

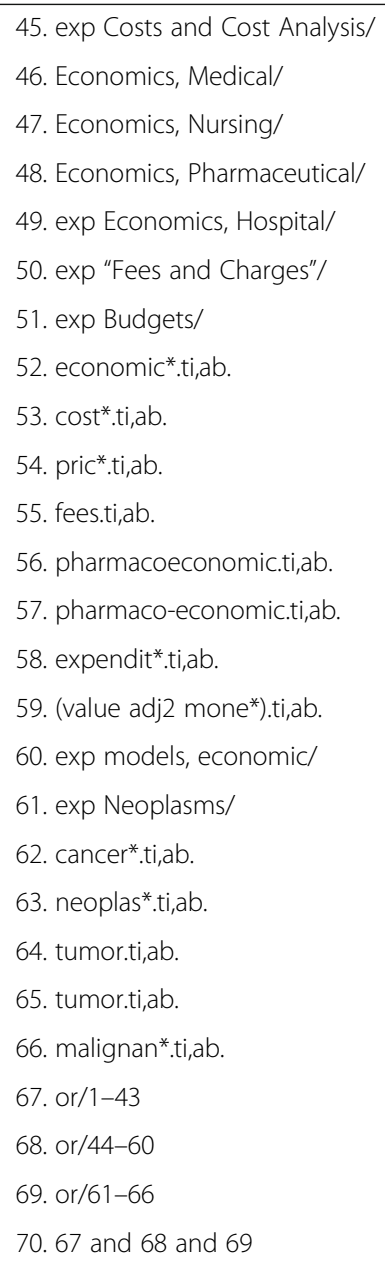

reported and analyzed) with hematologic and solid malignancies, where at least one treatment arm used one of the following immunotherapeutic agents, which were approved at the time of study design: -Anti-PD1 agents (nivolumab and pembrolizumab) -Anti-PDL1 agents (atezolizumab, avelumab, and durvalumab)

$\circ$ Anti-CTLA4 agents (ipilimumab and tremelimumab)

- Articles published in English between 1 January 2007 and 31 December 2018, inclusively. This period was chosen as it captures all reports on the above-mentioned therapeutic agents in human trials.

- Full text and original articles. Abstracts, conference proceedings, editorials, letters, reviews, and systematic reviews will be excluded. In the event of multiple publications reporting an analysis of the same underlying data, all articles meeting the inclusion criteria will be included in the analysis given the focus on methodological and reporting quality, rather than the conclusion reached by the underlying studies. 
Table 2 Data extraction form

\begin{tabular}{|c|c|c|}
\hline Variable name & Allowable values & Explanation \\
\hline \multicolumn{3}{|l|}{ Baseline trial characteristics } \\
\hline ID & Valid PubMed ID number & Pubmed ID \\
\hline Journal & Name of journal & Journal of publication \\
\hline Year & 2007-2018 & Year of publication \\
\hline Tumor site & $\begin{array}{l}\text { Non-small cell lung cancer Melanoma Renal cell } \\
\text { carcinoma Squamous cell carcinoma of the head } \\
\text { and neck Urothelial carcinoma Classical Hodgkin } \\
\text { lymphoma Other }\end{array}$ & $\begin{array}{l}\text { The tumor type for which treatment with a } \\
\text { check-point inhibitor was analyzed. }\end{array}$ \\
\hline \multirow[t]{7}{*}{ Check_point_1 } & Nivolumab & \multirow{7}{*}{$\begin{array}{l}\text { Name of the first check-point inhibitor } \\
\text { being compared. }\end{array}$} \\
\hline & Pembrolizumab & \\
\hline & Atezolizumab & \\
\hline & Avelumab & \\
\hline & Durvalumab & \\
\hline & Ipilimumab & \\
\hline & Tremelimumab & \\
\hline \multirow[t]{7}{*}{ Check_point_2 } & Nivolumab & \multirow{7}{*}{$\begin{array}{l}\text { Name of 2nd check-point inhibitor being } \\
\text { compared, if applicable. }\end{array}$} \\
\hline & Pembrolizumab & \\
\hline & Atezolizumab & \\
\hline & Avelumab & \\
\hline & Durvalumab & \\
\hline & Ipilimumab & \\
\hline & Tremelimumab & \\
\hline \multirow[t]{2}{*}{ Comparator_1 } & Placebo & \multirow{2}{*}{$\begin{array}{l}\text { Name of the } 1 \text { st non-check-point-inhibitor agent } \\
\text { or chemotherapeutic regimen being compared, } \\
\text { if applicable. }\end{array}$} \\
\hline & Name of other agent or regimen & \\
\hline \multirow[t]{2}{*}{ Comparator_2 } & Placebo & \multirow{2}{*}{$\begin{array}{l}\text { Name of the } 2 \text { nd non-check-point-inhibitor agent } \\
\text { or chemotherapeutic regimen being compared, } \\
\text { if applicable. }\end{array}$} \\
\hline & Name of other agent or regimen & \\
\hline Funding & Industry/government & Funding source declared by article \\
\hline Country & List of country names & $\begin{array}{l}\text { List of all the countries in which the economic } \\
\text { analysis applied as reported by the article. }\end{array}$ \\
\hline Multiple_countries & $\mathrm{Y} / \mathrm{N}$ & Did the economic analysis apply to multiple countries? \\
\hline \multicolumn{3}{|l|}{ CHEERS criteria } \\
\hline Title & $\mathrm{C} / \mathrm{l}$ & As detailed in CHEERS recommendations [9] \\
\hline Abstract & C/PC/I & As detailed in CHEERS recommendations [9] \\
\hline Background \& Objectives & C/PC/l & As detailed in CHEERS recommendations [9] \\
\hline Target population & $\mathrm{C} / \mathrm{PC} / \mathrm{l}$ & As detailed in CHEERS recommendations [9] \\
\hline Setting_Location & C/PC/I & As detailed in CHEERS recommendations [9] \\
\hline Perspective & C/PC/I & As detailed in CHEERS recommendations [9] \\
\hline Comparators & C/PC/I & As detailed in CHEERS recommendations [9] \\
\hline Time horizon & C/PC/I & As detailed in CHEERS recommendations [9] \\
\hline Discount rate & C/PC/l & As detailed in CHEERS recommendations [9] \\
\hline Health outcomes & C/PC/I & As detailed in CHEERS recommendations [9] \\
\hline Effectiveness & C/PC/I & As detailed in CHEERS recommendations [9] \\
\hline Preference for outcomes & C/PC/I/NA & As detailed in CHEERS recommendations [9] \\
\hline Resources and cost & $\mathrm{C} / \mathrm{PC} / \mathrm{l}$ & As detailed in CHEERS recommendations [9] \\
\hline Resource quantities & C/PC/I & As detailed in CHEERS recommendations [9] \\
\hline
\end{tabular}


Table 2 Data extraction form (Continued)

\begin{tabular}{lll}
\hline Variable name & Allowable values & Explanation \\
\hline Choice of model & $\mathrm{C} / \mathrm{PC} / \mathrm{I}$ & As detailed in CHEERS recommendations [9] \\
Assumptions & $\mathrm{C} / \mathrm{PC} / \mathrm{l}$ & As detailed in CHEERS recommendations [9] \\
Analytic methods & $\mathrm{C} / \mathrm{PC} / \mathrm{I}$ & As detailed in CHEERS recommendations [9] \\
Study parameters & $\mathrm{C} / \mathrm{PC} / \mathrm{I}$ & As detailed in CHEERS recommendations [9] \\
ICERs & $\mathrm{C} / \mathrm{PC} / \mathrm{I}$ & As detailed in CHEERS recommendations [9] \\
Uncertainty & $\mathrm{C} / \mathrm{PC} / \mathrm{l}$ & As detailed in CHEERS recommendations [9] \\
Heterogeneity & $\mathrm{C} / \mathrm{PC} / /$ NA & As detailed in CHEERS recommendations [9] \\
Limitations & $\mathrm{C} / \mathrm{PC} / \mathrm{l}$ & As detailed in CHEERS recommendations [9] \\
Funding source & $\mathrm{C} / \mathrm{I}$ & As detailed in CHEERS recommendations [9] \\
Conflict of Interest & $\mathrm{C} / \mathrm{I}$ & As detailed in CHEERS recommendations [9]
\end{tabular}

C complete; $P C$ partially complete; I incomplete; NA not applicable

Information sources, data management, and study selection A systematic search will be performed in Ovid MEDLINE ${ }^{\circledR}$ (1946 to present), OVID Embase (1974 to present), Costeffectiveness Analysis Registry (1926 to 2015), EvidenceBased Medicine Reviews, and NIHR-HTA (1989 to present) with the search strategy detailed in Table 1. Literature search results will be uploaded to EPPI-Reviewer 4 [19], in which two reviewers will independently assess the titles and abstracts of all articles retrieved from the database search. The full-text of articles potentially meeting the inclusion criteria will then be assessed for inclusion by the same two independent reviewers. Neither author will be blinded to the authors, institution, or journal of publication. Any disagreements will be resolved through discussion and consensus between the two reviewers, and a third reviewer will be asked to adjudicate on any cases where consensus cannot be reached. Data will then be extracted from all fulltext articles selected for analysis according to the data extraction form detailed in Table 2.

\section{Synthesis of results}

A qualitative description of the quality of included articles will be performed and data extracted (Table 2) will be provided in text and table form. All extracted data will be presented by article in table form. A random effects model will be used to assess the association of journal impact factor, year of publication, funding source, tumor site, trial or model-based study, and check-pointinhibitor studied on compliance with CHEERS criteria. The significance, magnitude, and confidence interval of associations will be presented in table form. Significance will be set at a two-sided $p$ value of $\leq 0.05$. All data will be analyzed using STATA 14 (StataCorp. 2015. Stata Statistical Software: Release 14. College Station, TX: StataCorp LP). As the current study focuses on the quality and reporting of economic analyses of CPIs, rather than the reported results, and the high heterogeneity expected of the included studies, no attempts will be made to quantitatively synthesize or analyze the results of the underlying studies, nor the risk of bias of the results, of the included articles. Wherever possible, all results will be presented in accordance with the PRISMA guidelines [20, 21].

\section{Discussion}

The described systematic review will aim to provide an overview of the quality of economic analyses on the use of novel check-point inhibitor therapies for the treatment of malignancies in adult patients. Any systemic or recurrent deficiencies in methodological or reporting quality will be described, and barriers to improvement explored, in an attempt to better characterize the quality of the evidence base on which financial toxicity assessments and funding policies are determined.

\section{Additional file}

Additional file 1: PRISMA-P 2015 checklist. (DOCX 33 kb)

\section{Abbreviations}

ASCO: American Society of Clinical Oncology; CHEERS: Consolidated Health Economic Evaluation Reporting Standards; CPIs: Check-point inhibitors; CTLA4: Cytotoxic T-lymphocyte-associated protein 4; FDA: Food and Drug Administration; ICER: Incremental cost-effectiveness ratio; ISPOR: International Society for Pharmacoeconomic and Outcomes Research; NIHR-HTA: National Institute for Health Research-Health Technology Assessment: PD1: Programmed death-1; PDL1: Programmed cell death ligand; PRISMA: Preferred Reporting Items for Systematic review and Meta-Analysis; PRISMA-P: Preferred Reporting Items for Systematic review and Meta-Analysis Protocols; PROSPERO: International prospective register of systematic reviews

\section{Acknowledgements \\ None.}

\section{Author contributions}

DS developed the search strategy. YW and PC drafted the manuscript, and developed and piloted the data extraction form. All authors contributed to the conception of the systematic review, development of the protocol, read, provided feedback and edits, and approved the final manuscript. 


\section{Funding}

This protocol and the planned study were not sponsored, and no funding has or will be received. YW receives fellowship funding from the University of British Columbia Clinical Investigator Program which was not involved in the development of the present protocol.

\section{Availability of data and materials}

The datasets generated and/or analyzed during the current study are available from the corresponding author upon reasonable request.

\section{Ethics approval and consent to participate}

Not applicable.

\section{Consent for publication}

Not applicable.

\section{Competing interests}

The authors declare that they have no competing interests.

\section{Author details}

${ }^{1}$ BC Cancer Vancouver, 600 West 10th Ave, Vancouver, British Columbia V5Z 4E6, Canada. ${ }^{2}$ Department of Medicine, Division of Community Internal Medicine, University of British Columbia, 2775 Laurel Street, 10th Floor, Vancouver, British Columbia V5Z 1M9, Canada. ${ }^{3}$ Department of Medical Oncology, CancerCare Manitoba, 675 McDermot Ave, Winnipeg, Manitoba R3E 0V9, Canada. ${ }^{4}$ Faculty of Health Sciences, Health Science Library, McMaster University, 1280 Main St W, Hamilton, Ontario L8S 4L8, Canada. ${ }^{5}$ Department of Oncology, McMaster University, Juravinski Cancer Centre, 3rd floor, 699 Concession Street, Hamilton, Ontario L8V 5C2, Canada.

Received: 24 April 2018 Accepted: 20 May 2019

Published online: 11 June 2019

\section{References}

1. Tang J, Shalabi A, Vm H-L. Comprehensive analysis of the clinical immunooncology landscape. Ann Oncol. 2018;29(1):84-91.

2. Postow MA, Sidlow R, Hellmann MD. Immune-related adverse events associated with immune checkpoint blockade. N Engl J Med. 2018; 378(2):158-68

3. Meropol NJ, Schrag D, Smith TJ, Mulvey TM, Langdon RM, Blum D, et al. American society of clinical oncology guidance statement: the cost of cancer care. J Clin Oncol. 2009;27(23):3868-74

4. Matter-Walstra K, Schwenkglenks M, Aebi S, Dedes K, Diebold J, Pietrini M, et al. A cost-effectiveness analysis of nivolumab versus docetaxel for advanced nonsquamous nsclc including pd-11 testing. J Thorac Oncol. 2016; 11(11):1846-55.

5. Vouk K, Benter U, Amonkar MM, Marocco A, Stapelkamp C, Pfersch S, et al. Cost and economic burden of adverse events associated with metastatic melanoma treatments in five countries. J Med Econ. 2016;19(9):900-12.

6. Tartari F, Santoni M, Burattini L, Mazzanti P, Onofri A, Berardi R. Economic sustainability of anti-pd-1 agents nivolumab and pembrolizumab in cancer patients: recent insights and future challenges. Cancer Treat Rev. 2016;48:20-4.

7. Gafni A, Birch S. Incremental cost-effectiveness ratios (icers): the silence of the lambda. Soc Sci Med. 2006;62(9):2091-100.

8. Khera N. Reporting and grading financial toxicity. J Clin Oncol. 2014;32(29): 3337-8

9. Husereau D, Drummond M, Petrou S, Carswell C, Moher D, Greenberg D, et al. Consolidated health economic evaluation reporting standards (cheers) statement. BMJ. 2013:346:f1049.

10. Husereau D, Drummond M, Petrou S, Carswell C, Moher D, Greenberg D, et al. Consolidated health economic evaluation reporting standards (cheers)--explanation and elaboration: a report of the ispor health economic evaluation publication guidelines good reporting practices task force. Value Health. 2013;16(2):231-50.

11. Meregaglia M, Cairns J. Economic evaluations of follow-up strategies for cancer survivors: a systematic review and quality appraisal of the literature. Expert Rev Pharmacoecon Outcomes Res. 2015;15(6):913-29.

12. Edwards K, Jones N, Newton J, Foster C, Judge A, Jackson K, et al. The costeffectiveness of exercise-based cardiac rehabilitation: a systematic review of the characteristics and methodological quality of published literature. Health Econ Rev. 2017;7(1):37.
13. lannazzo S, lliza AC, Perrault L. Disease-modifying therapies for multiple sclerosis: a systematic literature review of cost-effectiveness studies. Pharmacoeconomics. 2018;36(2):189-204.

14. Al Kadour A, Wa M, Al-Badriyeh D. Pharmacoeconomics evaluations of oral anticancer agents: systematic review of characteristics, methodological trends, and reporting quality. Value Health Reg Issues. 2018;16:46-60.

15. Aguiar PM, Lima TM, Storpirtis S. Systematic review of the economic evaluations of novel therapeutic agents in multiple myeloma: what is the reporting quality? J Clin Pharm Ther. 2016;41(2):189-97.

16. Monten C, Veldeman L, Verhaeghe N, Lievens $Y$. A systematic review of health economic evaluation in adjuvant breast radiotherapy: quality counted by numbers. Radiother oncol. 2017;125(2):186-92.

17. Shamseer $L$, Moher D, Clarke M, Ghersi D, Liberati A, Petticrew M, et al. Preferred reporting items for systematic review and meta-analysis protocols (prisma-p) 2015: elaboration and explanation. BMJ. 2015:350:g7647.

18. Moher D, Shamseer L, Clarke M, Ghersi D, Liberati A, Petticrew M, et al. Preferred reporting items for systematic review and meta-analysis protocols (prisma-p) 2015 statement. Syst Rev. 2015;4(1):1.

19. Thomas J, Brunton J, Graziosi S. Eppi-reviewer 4.0: software for research synthesis. London: Social science research unit, Institute of education, University of london; 2010. Available from: https://eppi.ioe.ac.uk/cms/default aspx?tabid=1913. Accessed 31 May 2019.

20. Moher D, Liberati A, Tetzlaff J, Altman DG, Prisma Group. Preferred reporting items for systematic reviews and meta-analyses: the prisma statement. J Clin Epidemiol. 2009;62(10):1006-12.

21. Liberati A, Altman DG, Tetzlaff J, Mulrow C, Gøtzsche PC, JPA I, et al. The prisma statement for reporting systematic reviews and meta-analyses of studies that evaluate health care interventions: explanation and elaboration. J Clin Epidemiol. 2009;62(10):e1-34.

\section{Publisher's Note}

Springer Nature remains neutral with regard to jurisdictional claims in published maps and institutional affiliations.
Ready to submit your research? Choose BMC and benefit from:

- fast, convenient online submission

- thorough peer review by experienced researchers in your field

- rapid publication on acceptance

- support for research data, including large and complex data types

- gold Open Access which fosters wider collaboration and increased citations

- maximum visibility for your research: over $100 \mathrm{M}$ website views per year

At $\mathrm{BMC}$, research is always in progress.

Learn more biomedcentral.com/submissions 